\title{
REALIDADE VIRTUAL E ROBÓTICA EM CIRURGIA-AONDE CHEGAMOS E PARA ONDE VAMOS?
}

\author{
VIRTUAL REALITY AND ROBOTICS IN SURGERY: WHERE WE ARE AND WHERE WE \\ ARE HEADING
}

\author{
James Skinovsky, TCBC-PR'; Maurício Chibata, TCBC-PR²; \\ Daniel Emilio Dalledone Siqueira ${ }^{3}$
}

\section{INTRODUÇÃO}

Cirurgia Minimamente Invasiva, Telecirurgia, Robótica e Realidade Virtual; estas fronteiras tecnológicas têm revolucionado a prática operatória na atualidade. Toda nova tecnologia visa a melhora da qualidade de atendimento aos nossos pacientes, mas demanda da classe médica a saída da inércia científica, treinamento, gasto de tempo e dinheiro e estresse. Os cirurgiões já lideraram no passado grandes revoluções médicas, como a introdução da antissepsia por Semelweiss, o uso da anestesia por Warren, a antibioticoterapia, os transplantes e o início da cirurgia minimamente invasiva por Mouret e Perissat. Estamos no limiar de uma nova revolução onde combinaremos mínimos acessos, informática, robótica e teletransmissão?

Com a introdução dos robôs na área cirúrgica, no início da década de 80 , numerosas promessas tornaram-se promissoras, a maioria das quais não adquiriu importância na prática operatória regular. $\mathrm{O}$ advento da cirurgia minimamente invasiva levou ao mesmo tempo à melhora das aptidões robóticas e ao aparecimento do promissor campo do treinamento em realidade virtual. Os investimentos levaram ao desenvolvimento de diversos programas e sets de treinamento virtual e de 3 sistemas robóticos: AESOP ${ }^{\circledR}$, Zeus ${ }^{\circledR}$ e da Vinci ${ }^{\circledR}$; nenhum deles recebeu a designação de robô pelo FDA ( Food Drugs Administration) americano e nenhum é capaz de realizar tarefas cirúrgicas pré-programadas, a definição oficial de um robô, mas novas tecnologias se desenvolverão e um mundo novo deve abrir-se às portas de nossa realidade cirúrgica.

Esta revisão visa levar ao conhecimento dos que labutam na área operatória o que já foi feito, onde estamos e para onde este fascinante mundo nos levará.

\section{1 - Realidade Virtual e Cirurgia}

Realidade Virtual (RV) é uma tecnologia gerada por computação que disponibiliza informação com o objetivo de simular a vida em seu meio ambiente natural.

Apesar de soar aos nossos ouvidos como um filme de ficção científica, esta tecnologia já está ao nosso redor há vários anos; desde os anos 40, programas de simulação têm sido utilizados para avaliar e certificar pilotos da aviação militar e comercial. Durante os últimos anos, a RV tem avançado para o campo da medicina, prometendo tornar -se nos tempos que virão, a próxima grande revolução no ensino médico.

Os primeiros movimentos no sentido da aplicação do mundo virtual no campo cirúrgico aconteceram na primeira metade da década de 90, já então anunciados pelo profeta da tecnologia médica, Richard Satava, em seu estudo "Robotics, telepresence and virtual reality: a critical analysis of the future of surgery", publicado em 1992.

No treinamento cirúrgico, em decorrência de problemas jurídicos, os aprendizes têm pouca oferta de operações para treinamento, levando à prática deficiente e à queda na auto-confiança. Com o aumento do grau de dificuldade dos procedimentos operatórios ofertados no mundo moderno, torna-se imperativo que os aprendizes tenham uma arena para seu aperfeiçoamento; com o advento desta tecnologia, isto tornou-se realidade.

Diversos estudos ao redor do globo tem demonstrado que as habilidades a serem adquiridas pelos cirurgiões que se iniciam em vídeocirurgia são realizadas de maneira mais rápida com o uso dos simuladores cirúrgicos, pois além da exaustiva repetição, os treinandos são avaliados pelo próprio programa, fazendo com que suas imperfeições sejam reconhecidas e corrigidas de maneira efetiva ${ }^{2-9}$.

Os atuais simuladores já permitem que o treinando experimente a sensação tátil individualizada por tecido, o chamado biofeedback, aproximando ainda mais a simulação da realidade ${ }^{10}$.

Aplicações Atuais da Realidade Virtual em Cirurgia:

- Aprendizado em modelos anatômicos virtuais;

- Simulação em treinamento cirúrgico: A primeira aplicação desta tecnologia em cirurgia videolaparoscópica foi em modelo de treinamento para colecistectomia; desde então variados modelos de sets de treinamento e de tarefas foram sendo disponibilizados, discutidos e avaliados.

Já existem no mercado simuladores para: Cirurgias ortopédicas (Projeto Hipócrates); procedimentos

1. Doutor em Clínica Cirúrgica pela UFPR; Professor Titular de Clínica Cirúrgica do UnicenP.

2. Mestre em Clínica Cirúrgica pela UFPR.

3. Acadêmico de Medicina do UnicenP

Recebido em 21/12/2007

Aceito para publicação 27/02/2008

Conflito de interesse: nenhum

Fonte de financiamento: nenhuma 
endoscópicos variados, envolvendo cirurgia vascular, urologia e o aparelho digestório (endoscopia digestiva alta e baixa diagnóstica e terapêutica, colangiopancreatografia retrógrada endoscópica, coledocoscopia etc.); Cirurgias videolaparoscópicas, cuja simulação inclui: tarefas básicas sutura (Figuras 1 e 2), hemostasia, dissecação, etc.; colecistectomia; gastrofundoplicatura; hernioplastia inguinal, incisional; gastroplastia e outras.

Esta inovadora tecnologia apresenta marcadas vantagens, como:

Eficiência - elimina o risco de lesões inadvertidas em pacientes reais, durante o desenrolar da curva de aprendizado inicial, prevenindo problemas jurídicos decorrentes;

Objetividade - a realidade virtual pode objetivamente avaliar e medir a competência técnica, bem como sua evolução, levando inclusive à melhoria da auto-estima cirúrgica;

Ética - a técnica permite a exaustiva repetição inicial, fazendo com que o treinando suba os próximos degraus (modelos animais, humanos), já com maior segurança e habilidade.

É menos ofensiva do que treinar em modelos animais, podendo, com o passar do tempo, tornar-se menos onerosa.

Apresenta como desvantagem o custo, pois novas tecnologias são caras, mas a tendência de barateamento ocasionado pela concorrência e pelo desenvolvimento tecnológico é irrefreável.

\section{2 - Robótica e Cirurgia}

O uso da robótica tem se desenvolvido nos últimos 75 anos, mas somente nos últimos cinco anos o uso potencial da chamada mecatrônica no campo cirúrgico tem chamado a atenção da comunidade científica mundial.

Nas últimas décadas, os robôs vem apresentando lugar de destaque no campo da ficção científica; suas descrições variam de máquinas que monotonamente repetem movimentos, como citado pelo escritor tcheco Capek, em 1921, ao ultra-inteligente robô de Issac Asimov nos anos 50, indo ao encontro dos familiares R2D2 e C3PO de Guerra nas Estrelas e do incrível ciborg de Exterminador do Futuro.

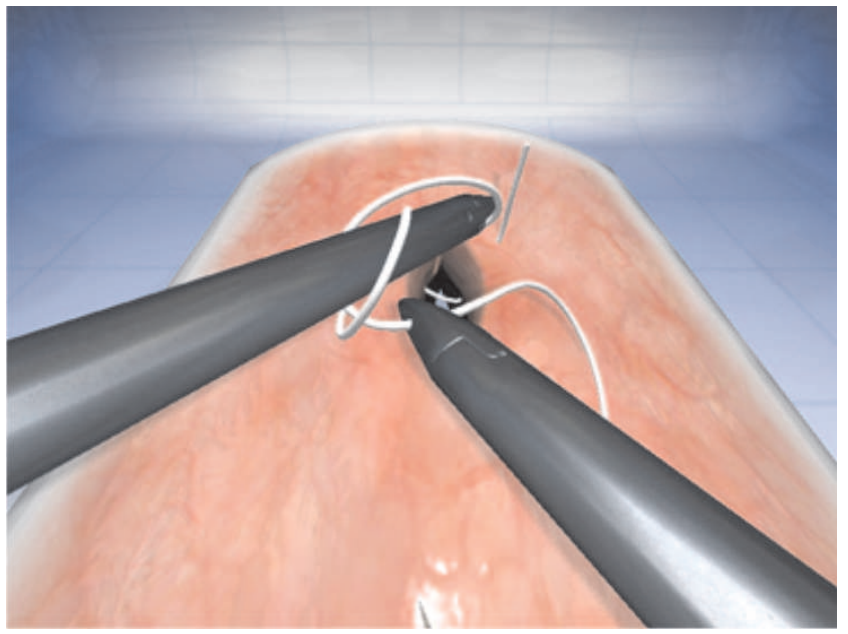

Figura 1 - Simulação de nó intra-corpóreo - Simulador Simbionix ${ }^{\circledR}$.
Os robôs gradualmente ocuparam seu espaço em nosso mundo, realizando tarefas repetitivas, perigosas ou que exijam grande precisão, como na indústria automobilística ou em testes nucleares.

Enquanto muitos poderiam exceder a performance humana em tarefas específicas, nenhum adquiriu sequer inteligência comparável a um bebê de dois anos de idade nunca demonstrando habilidades cognitivas ${ }^{11}$.

Os recentes conceitos de robôs cirurgiões iniciaram com os trabalhos de Scott Fisher, da NASA, na segunda metade da década de 80, cujo grupo desenvolveu paralelamente conceitos de realidade virtual, imagens 3D e suas interfaces ${ }^{11-13}$.

Nos estertores da década de 80, o desenvolvimento paralelo da videocirurgia emergiu no campo cirúrgico. Jacques Perissat apresentou vídeo de uma colecistectomia laparoscópica no congresso da SAGES americana (Society of American Gastrointestinal Endoscopic Surgeons) causando profundo impacto no conceito "grandes cirurgiões, grandes incisões".

Logo em seguida, Richard Satava e um time da NASA começaram a desenvolver, sob a chancela de programa militar do pentágono, programa de cirurgia por telepresença, simplesmente resumido por cirurgia à distância ${ }^{11}$.

No início da década de 90, diversos sistemas robóticos cirúrgicos começaram a ser desenvolvidos, como o RoboDoc ${ }^{\circledR}$, capaz de realizar anastomoses vasculares e o Artemis $^{\circledR}$, com manipulação à distância ${ }^{10}$.

Em 1992, os militares americanos desenvolveram o programa DARPA ${ }^{\circledR}$, com o objetivo de salvar soldados no campo de batalha, utilizando alta tecnologia. Seu uso combinava sensoreamento remoto, robótica, telemedicina e realidade virtual, através da utilização de um veículo militar comandado por controle remoto e equipado com exames de imagem de última geração, onde, uma vez constatada a necessidade, os procedimentos cirúrgicos seriam iniciados por telecirurgia robótica $^{11,12}$.

Em 1993, Alberto Roveda realizou biópsia hepática em suíno, com a estação cirúrgica situada no laboratório da NASA, em Pasadena, Califórnia, estando o animal operado do outro

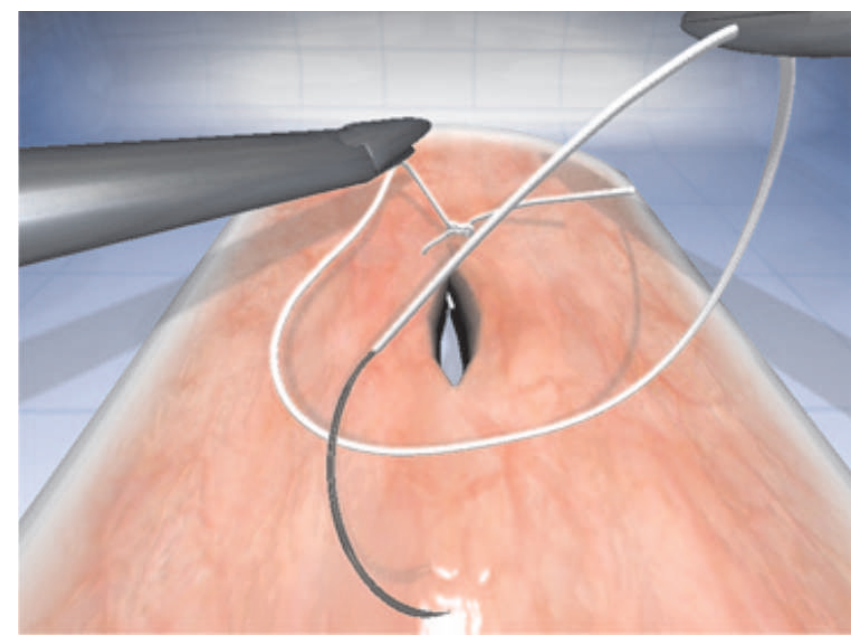

Figura 2 - Simulação de nó intra-corpóreo - Simulador Simbionix ${ }^{\circledast}$. 
lado do Atlântico, em Milão (o delay foi de 1,2 seg) - a sensação tátil e o biofeedback, foram utilizados nesta experiência ${ }^{11,12}$.

A primeira aplicação comercial da robótica na manipulação cirúrgica direta aconteceu com o $\mathrm{AESOP}^{\circledR}$, braço mecânico que utilizava comando de voz para o controle do movimento da óptica em videocirurgia ${ }^{11}$.

Logo em seguida foram desenvolvidos para uso comercial os sistemas cirúrgicos Zeus ${ }^{\circledR} \mathrm{e} \mathrm{daVinci}^{\circledR}$ (Figura 3) e, em abril de 1997, foi realizada a primeira cirurgia robótica em paciente, na cidade de Bruxelas, por Jacques Himpens e Cardiere ${ }^{15}$.

Em 2001, o Zeus ${ }^{\circledR}$ foi utilizado em uma cirurgia transatlântica entre Nova York e Estrasburgo (França), por Marescoux e Gagner ${ }^{16,17}$ com delay de 155 mseg. É importante ressaltar que atrasos maiores que $200 \mathrm{mseg}$ inviabilizam as operações feitas com grandes distâncias, pois o tecido pode se mover antes da percepção de movimento do cirurgião, podendo causar lesões inadvertidas).

\section{3 - Olhando para o futuro}

Assim como os simuladores são agora os parâmetros rotineiros de treinamento utilizados no campo da aviação e atividade aeroespacial, logo também o serão na área médica. Estes equipamentos permitirão cada vez mais a instrução da correta técnica operatória sem a necessidade da utilização de pacientes vivos, pelo menos nos estágios iniciais do treinamento.

A futura geração das imagens virtuais permitirá a clara visualização da anatomia cirúrgica em tempo real, através da interface com imagens orgânicas individuais armazenadas previamente à cirurgia, definindo planos de dissecação conforme a anatomia interna do indivíduo, em três dimensões. É a chamada Navegação Cirúrgica Virtual.

Em 1998, no artigo intitulado "Virtual Reality Applied to Hepatic Surgery Simulation: The Next Revolution"18, publicado no Annals of Surgery, por Marescoux et al., previa: "Usando conceitos de realidade virtual (navegação, interação e imersão),

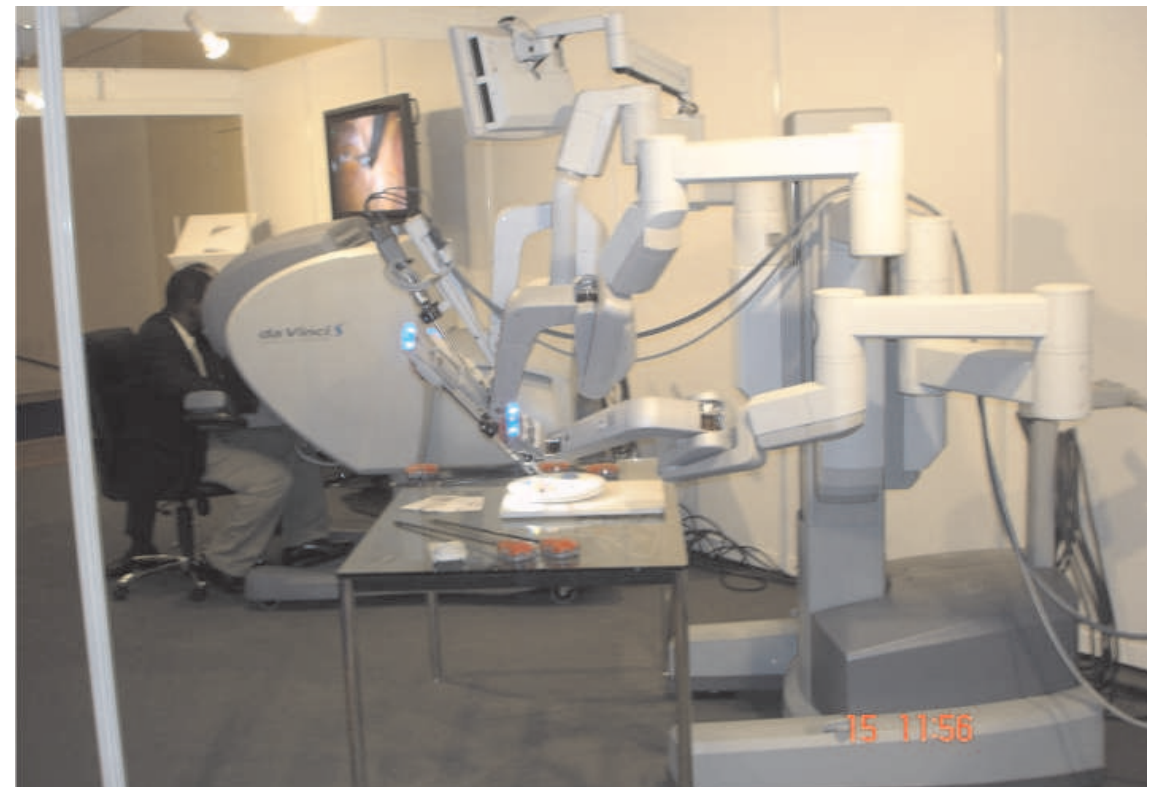

Figura 3 - Set de treinamento do sistema robótico daVinci ${ }^{\circledR}$.

planejamento cirúrgico e treinamento, o ensino e o aprendizado de procedimentos complexos pode ser plenamente possível. A habilidade de praticar o gestual cirúrgico repetidamente irá revolucionar o treinamento, e a combinação de planejamento operatório e simulação melhorará a eficiência da intervenção, levando ao ótimo aproveitamento à causa do paciente".

Oito anos depois, em 2006, o "futuro" foi demonstrado por Marescoux, no X Congresso Mundial de Cirurgia Endoscópica, em Berlim.

Com o advento da realidade virtual, os cirurgiões terão a oportunidade de aprender e praticar as habilidades inerentes à sua área de trabalho, quantas vezes quiserem e onde o desejarem, bastando para isso um programa de computador, uma tela e um jogo de joysticks.

A próxima geração da internet, com fibras ópticas de $45 \mathrm{Mbyte} / \mathrm{seg}$ universalmente distribuídas, podem tornar a cirurgia remota realidade em diversos locais no planeta.

Os sistemas atuais são somente o início da revolução robótica, pois o futuro próximo vislumbra uma única estação de trabalho, comandada pelo cirurgião, onde imagens pré-operatóri- as do paciente podem ser acessadas durante o procedimento, permitindo a navegação virtual em tempo real e a perfeita visualização anatômica trans-operatória. Além disso, o sistema controlará o meio ambiente da sala cirúrgica (temperatura, luz, etc.), o uso e potência instrumental (cautérios, bisturi ultrassônico) e o sistema de comunicação com o exterior (telemedicina), o que podemos chamar de "Centro Cirúrgico do Futuro".

A evolução intelectual dos robôs deverá permitir que partes ou todo o ato cirúrgico seja programado previamente e realizado por eles, superando as limitações físicas humanas, como fadiga muscular e nossos limitados movimentos articulatórios.

A diminuição do tamanho dos equipamentos e de seu custo e a nanotecnologia, fazendo com que pequenos robôs trabalhem no interior de nosso corpo, devem revolucionar ainda mais a Medicina nos tempos vindouros.

A ilimitada capacidade humana de dar forma aos seus sonhos, de unir o futuro ao presente, de buscar o intangível, é o que nos traz a certeza de que nenhum devaneio é impossível e de que o tempo vindouro na verdade inicia-se daqui a um minuto. Bem-vindo ao futuro! 


\begin{abstract}
Minimally Invasive Surgery, Telesurgery, Robotics and Virtual Reality represent the technological frontiers that have revolutionized operating practices nowadays. These new technologies aim at improving the quality of assistance offered to patients; thus, they demand from the medical staff more effective measures as far as scientific research, training and expenditure of time and financial resources are concerned. In the past, surgeons have led several medical revolutions, such as the use of antiseptic surgical methods by Semelweiss, the use of anesthesia by Warren, antibiotic therapy, the transplants and the onset of the minimally invasive surgery by Mouret and Perissat. The objective of this article is to present the outreach of this new technology which comprises minimal access, computing, robotics and teletransmission. We have concluded that the new technologies developed in the medical field in the last decades, will offer new options and challenges for the treatment of the surgical patient, leading the scientific knowledge to a new era, the one of the virtual environment (Rev. Col. Bras. Cir. 2008; 35(5): 334-337).
\end{abstract}

Key words: Robotics; Specialties, Surgical.

\section{REFERÊNCIAS}

1. Satava RM. Robotics, telepresence and virtual reality: a critical analysis of the future of surgery. Minimally Invasive Therapy. 1992;1:357-63

2. Soler L, Ayach N, Nicolau S, Pennec X, Forest C, Delingette H, Mutter D, Marescoux J. Virtual reality, augmented reality and robotics in digestive surgery. World Scientific Publisher Edition. 2004; pp476-484.

3. Raibert M, PlayterR, Krummel,TM. The use of a virtual reality haptic device in surgical training. Acad Med.1998;73:596-97.

4. Ota D, Loftin B, Saito T, Lea R, Keller J. Virtual reality in surgical education. Comput Bio Med.1995; 25(2):127-137.

5. Ahlberg G, Heikkinen T, Leijonmarck CE, Rutqvist J, Arvidsson D. Does training in a virtual reality simulator improve surgical performance? Surg Endosc.2002;16(1):126-129.

6. Grantcharov TP, Rosenberg J, Pahle E, Funch-Jensen P. Virtual reality computer simulation. Surg Endosc.2001;15(3):242-244.

7. Woodman R. Surgeons should train like pilots. Br Med J.1999; 319:1312.

8. Gallagher AG, McClure N, McGuigan J, Crothers I, Browning J. Virtual reality training in laparoscopic surgery: a preliminary assessment of minimally invasive surgical trainer virtual reality. Endoscopy.1998; 30:617-620.

9. Cushieri A. Visual displays and visual perception in minimal access surgery. Seminars in laparoscopic surgery.1995;2:209-14.

10. Satava RM. Virtual reality and telepresence for military medicine. Comput Biol Med.1995;2:229-36.

11. Satava RM. Surgical robotics: the early chronicles: a personal historic perspectives. Surg Laparosc Endosc Perc Tech. 2002;12: 6-16.
12. Wihelm DM, Ogan K, Roehrborn CG, Cadeddu JA, Pearle MS Assesment of basic endoscopic performance using a virtual reality simulator. J Am Coll Surg.2002; 195(5):675-681.

13. Wang Y, Sackier J. Robotically enhanced surgery: from concept to development. Surg. Endosc.1994;8:63-66.

14. Wickham JEA. Future developments of minimally invasive therapy. Brit Med. J.1995;308 -193-6.

15. Himpens J, Leman G, Cardiere, GB. Telesurgical laparoscopic cholecystectomy. Surg Endosc.1998;12:1091.

16. Marescoux J, Leroy J, Gagner M, Rubino F, Mutter D, Vix M, Butner SE, Smith MK. Transatlantic robot-assisted telesurgery. Nature. 2001; 413:379-80.

17. Anvari M, McKinley C, Stein H. Establishment of the world's first telerobotic remote surgical service. Ann Surg. 2005;241:460-4.

18. Marescoux J, Clement JM, Tasseti V, Koehl C, Sotin S, Russier Y, Mutter D, Delingete H, Ayache N. Virtual reality applied to hepatic surgery simulation: the next revolution. Ann Surg.1998;228:627-34.

Como citar este artigo:

Skinovsky J, Chibata M, Siqueira DE. Realidade virtual e robótica em cirurgia - Aonde chegamos e para onde vamos? Rev Col Bras Cir. [periódico na Internet] 2008; 35(5). Disponível em URL: http:// www.scielo.br/rcbc

Endereço para correspondência:

James Skinovsky

R. Arion Niepce da Silva,120 - apto.116 - Portâo

80610-310 - Curitiba - PR

Tel: (41)9971-1644

E-mail: jameskk@uol.com.br 